\title{
High resolution of a small pericentric inversion of chromosome 11
}

\author{
HELENA AUTIO-HARMAINEN AND ALBERT DE LA CHAPELLE \\ From the Department of Medical Genetics, University \\ of Helsinki, and Folkhälsan Institute of Genetics, Helsinki, Finland
}

SUMMARY A pericentric inversion 11 (p11q13.3) segregating in two generations is described. A high $\vec{\nabla}$ degree of resolution of the inversion was achieved by using prophase and prometaphase chromosomes from methotrexate-synchronised cells. The inversion occurred in a mother and three of her $\vec{A}$ ten children. It had no detectable clinical consequences.

With better methods of chromosomal banding, increasing numbers of pericentric inversions are being detected. Some inversions do not appear to carry any appreciably increased risk of unbalanced offspring ${ }^{1}$ and may even be referred to as harmless variants (for example, the common inversion in chromosome 9). Heterozygotes for other inversions have produced unbalanced offspring. ${ }^{2}$ It has been proposed that 'large' inversions are more likely than 'small' ones to produce unbalanced offspring, ${ }^{3}$ but exceptions to this rule do occur. Careful reporting of adequately studied cases can help shed light on this problem.

We report here a previously unrecorded inversion of chromosome 11 which could be accurately characterised by the use of prophase and prcmetaphase mitoses. ${ }^{4}$ The inversion is compared to three previously reported pericentric inversions of chromosome $11 . .^{5}$

\section{Methods}

Lymphocytes were cultured and harvested by standard methods. To one portion of each sample, methotrexate (Lederle) was added at a concentration of $10^{-7} \mathrm{M}$ after 72 hours' incubation at $37^{\circ} \mathrm{C}$. The methotrexate block was released 17 hours later by the addition of thymidine at a concentration of $10^{-5} \mathrm{M}$. The cells were then allowed to grow at $37^{\circ} \mathrm{C}$ for 5 to $5 \frac{1}{2}$ hours and treated with colcemid during the final 15 minutes. This was a modification of the methods described by Yunis ${ }^{4}$ and Yunis et al. ${ }^{7}$ Slide preparations were made and air-dried for 24 hours. G-banding, ${ }^{8}$ C-banding, ${ }^{9}$ and R-banding ${ }^{10}$ were used.

Received for publication 28 March 1979

\section{Results}

The proband was a 24-year-old woman married for 5 years to a healthy man. She was referred for ${ }_{\infty}^{\infty}$ chromosome analysis as a routine part of a thorough. clinical investigation for primary sterility. There had응 been no miscarriages. She was markedly obeses and was repeatedly found to have a high level of plasma testosterone. The hormonal inbalance wasڤ thought to be the most probable cause of her 2 sterility.

The proband had inherited the inverted chromosome from her mother (fig 1) who had had 105 children, all healthy, and no miscarriages. Two of the proband's brothers had died in accidents. The remaining 7 sibs were karyotyped; two of these were inversion heterozygotes and five were not. The wiveso of the two brothers with the inversion had not had any miscarriages. The proband and her twin sistero were dizygotic; they resembled each other very little. The proband's husband was cytogeneticallyo normal.

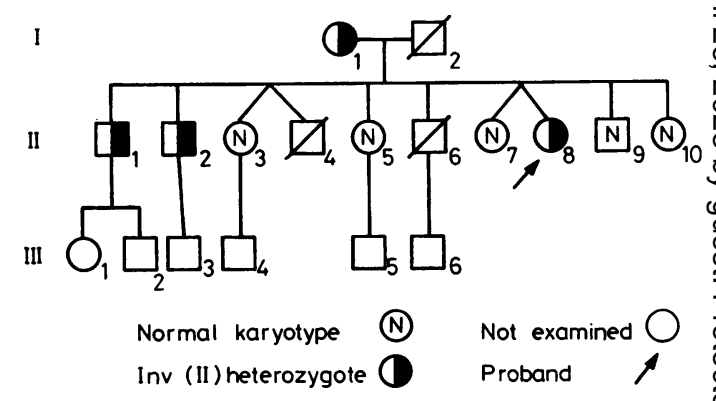

FIG 1 Pedigree of family. 


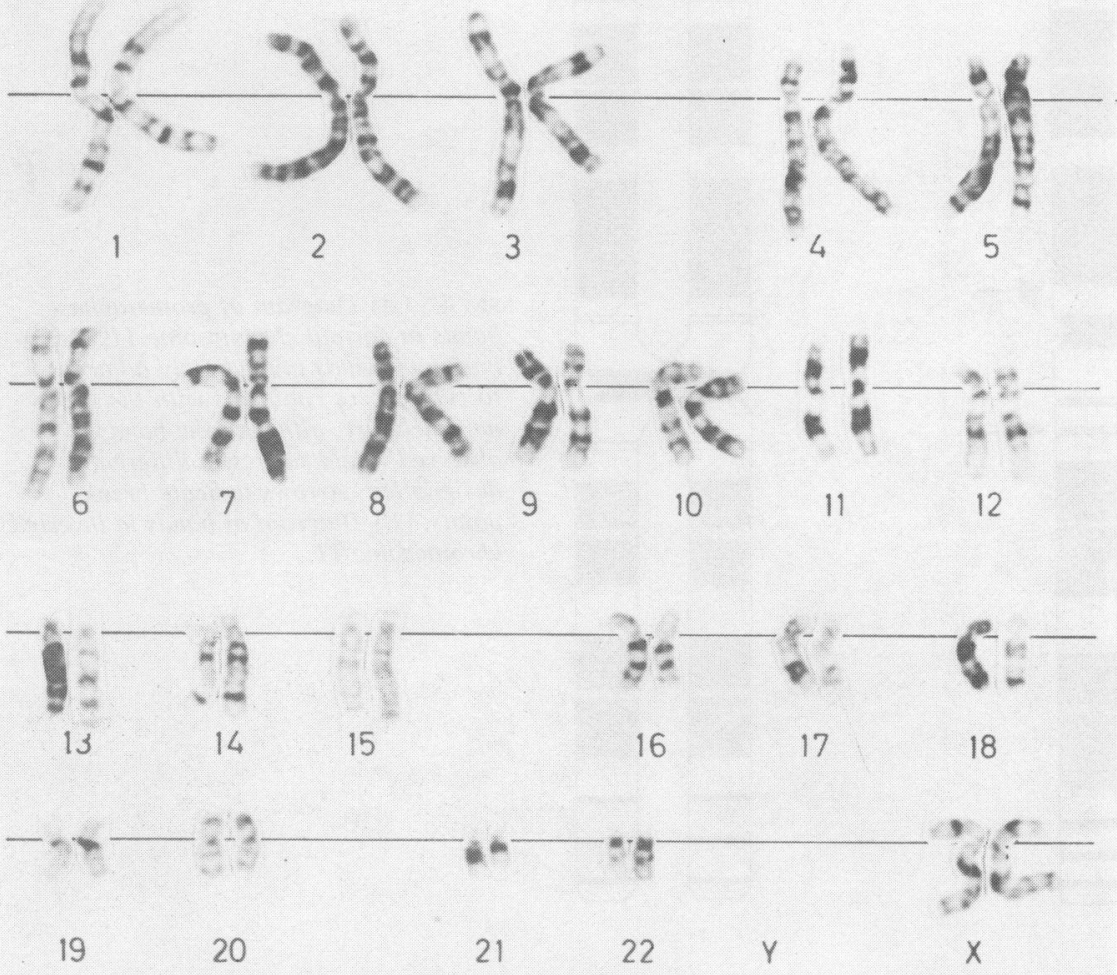

FIG 2 Karyotype of proband. Note metacentric appearance of inverted chromosome 11 (right)

The abnormality in one of the chromosomes 11 was first recognised in ordinary mid-metaphase mitoses by its almost metacentric appearance (fig 2). The breakpoints were difficult to determine from such cells, but prophase and prometaphase chromosomes were very useful (fig 3 ). The lightly staining band referred to as q13 in the Paris nomenclature had at least two fine dark bands detectable by high resolution techniques (fig 4A), ${ }^{4}$ while no such 'new' dark band was seen in the light band on the short arm side of the centromere p11. In favourable cells it could be clearly seen that the more proximal of the faint dark bands in q13 (q13.2) was displaced to the short arm, while the more distal one (q13.4) remained in the long arm. The inversion could thus be written inv(11) (p11q13.3) as illustrated in the diagram (fig 4B). Thus, even though this was a small pericentric inversion involving $15 \%$ of the chromosome or less, its effect on the position of the centromere (more

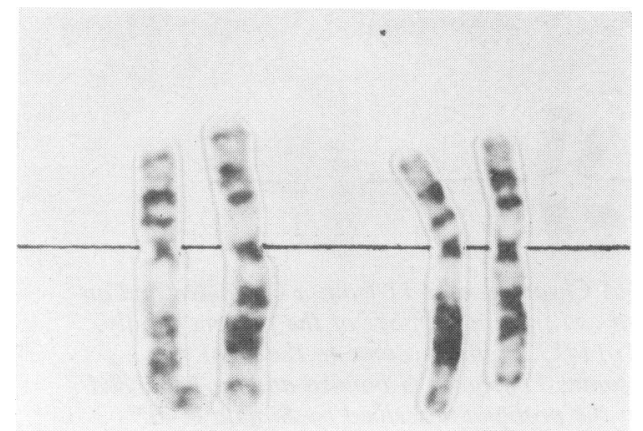

FIG 3 Prometaphase chromosomes 11 from two cells of proband. Inverted chromosome is to the right.

metacentric) was so definite that it was detectable at mid-metaphase. R-banding corroborated the above interpretation. In the right-hand (inverted) 

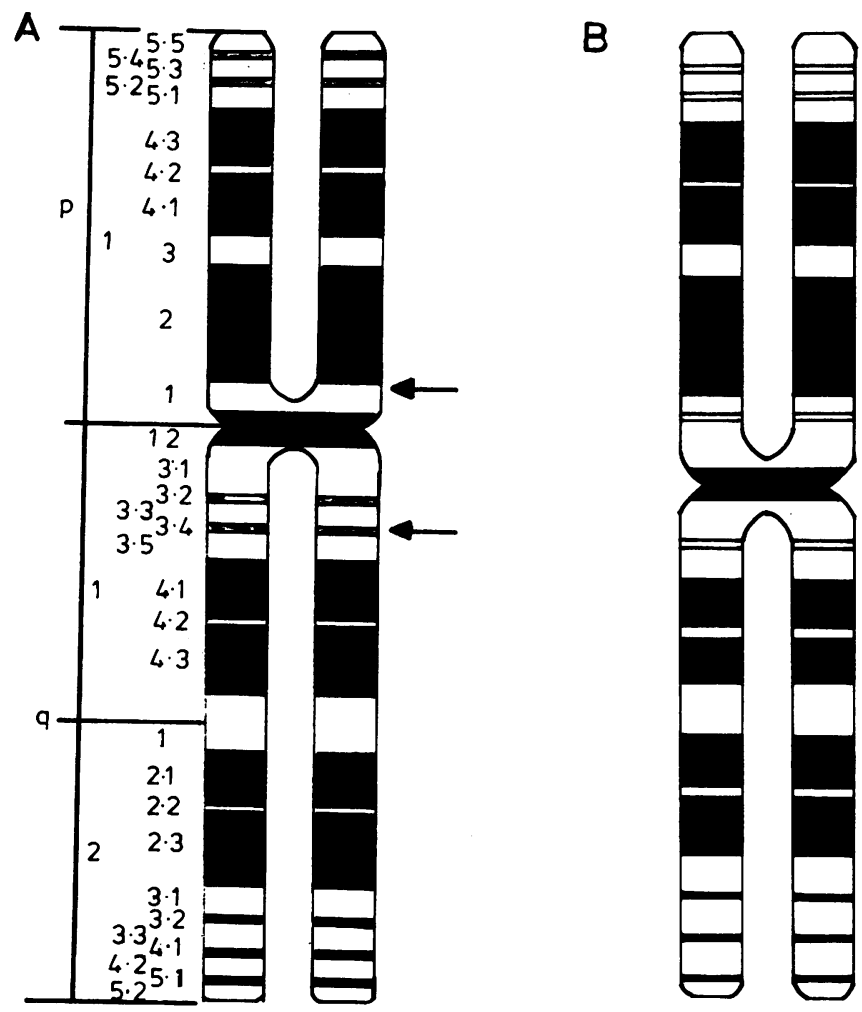

FIG 4 (A) Diagram of prometaphase bands in normal chromosome 11. Designation of bands immediately adjacent to centromere conforms with the Paris nomenclature, although the bands observed would suggest a different designation. Arrows indicate breakpoints. (B) Diagram of bands in inverted chromosome 11.

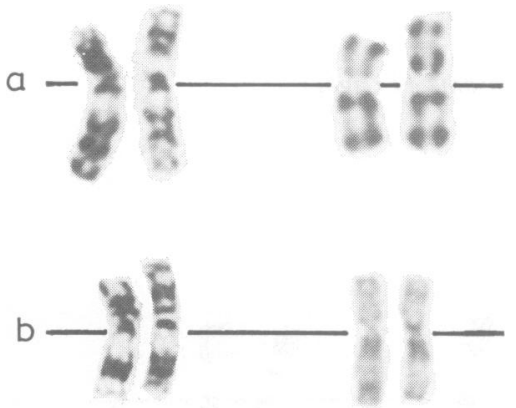

FIG 5 (a) Chromosomes 11 from a G-banded and an $R$-banded cell from a member of the present family. Inv(11) (p11q13.3) chromosome to the right. (b) Chromosomes 11 from a G-banded and an R-banded cell from the proband described by Simola et al. ${ }^{5}$ $\operatorname{Inv}(11)$ (p15q23) chromosome to the right.

chromosome in fig $5 \mathrm{a}$ the major feature is as expected: the dark band q13 is smaller and the dark band p11 correspondingly larger in the inverted chromosome.

C-banding showed no abnormality. The inverted chromosome could be identified even without $\overrightarrow{\overrightarrow{0}}$ sequential banding by its metacentric appearance. Its $\mathbf{C}$ band was located in the middle of the centro $=\frac{7}{0}$ mere as expected.

\section{Discussion}

We are aware of three other pericentric inversions of chromosome 11, namely an inv(11)(p11q11) detectable by C-banding only, ${ }^{5}$ an inv(11)(p15q14) described by Boué and Boué, ${ }^{6}$ and an inv(11) (p15q23) described in this laboratory. ${ }^{5}$ The first twoo are quite different and can hardly be confused with the present one. However, the third greatly resembles $N$ the present one in appearance, since both lead to a N nearly metacentric chromosome 11 whose major bands appear undisturbed. In spite of the apparent ${ }^{\omega}$ similarities, the breakpoints have been interpreted considerably differently. According to Simola et al ${ }^{50}$ their inversion comprised most of the chromosome (p15q23), while the present one is small ${ }^{+}$ (p11q13). Since it is of considerable interest to ${ }_{0}^{\circ}$ establish the extent of the inverted segment in each reported case we re-examined the chromosomes of $\frac{\text { ? }}{\mathrm{D}}$

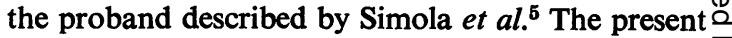


findings (fig $5 \mathrm{~b}$ ) support the earlier interpretation. Note, for instance, the R-banded chromosomes at the extreme right in each line. In the lower line (the patient of Simola et al) the dark bands in the end regions (p15 and q23) are affected but the pericentromeric bands are unaffected, while in the upper line (present proband) the pericentric dark bands are of altered appearance with respect to the normal homologue. We conclude that in the present family a very small, and in the family described by Simola et $a l,{ }^{5}$ a very large pericentric inversion is segregating.

From the pedigree it can be deduced that the present inversion carries little risk for unbalanced offspring or even miscarriages. This knowledge may possibly be used in the clinical handling and counselling of this family. However, even though 'large' inversions tend to carry a higher risk for unbalanced offspring than 'small' ones, ${ }^{3}$ we confirm here the presence of a large inv(11) in a previously described family with no malformed children. ${ }^{5}$ Conversely, 'small' inversions have occasionally produced unbalanced offspring. While this is biologically quite understandable, it implies that all newly detected pericentric inversions must be regarded as potentially harmful. Only careful family studies in each case can give a clue to the likely outcome of meiosis and gametogenesis in the individual carrier.

This work was supported by grants from the Sigrid Jusélius Foundation and the Nordisk Insulinfond.

\section{References}

1 de la Chapelle A, Schröder J, Stenstrand K, Fellman J, Herva R, Saarni M, Anttolainen I, Tallila I ,Tervilä L, Husa L, Tallqvist C, Robson EB, Cook PJL, Sanger R. Pericentric inversions of human chromosomes 9 and 10. Am J Hum Genet 1974;26:746-66.

2 Moorhead PS. A closer look at chromosomal inversions. Editorial. Am J Hum Genet 1976;28:294-6.

3 Winsor EJT, Palmer CG, Ellis PM, Hunter JLP, FergusonSmith MA. Meiotic analysis of a pericentric inversion, inv(7) (p22q32), in the father of a child with a duplicationdeletion of chromosome 7. Cytogenet Cell Genet 1978; 20:169-84.

4 Yunis JJ. High resolution of human chromosomes. Science 1976;191:1268-70.

5 Simola K, Karli P, de la Chapelle A. Two pericentric inversions of human chromosome 11. J Med Genet $1977 ; 14: 371-4$

- Boué J, Boué A. Prenatal diagnosis in 100 structural rearrangements of the chromosomes. Cytogenet Cell Genet 1978;20:213-25.

7 Yunis JJ, Sawyer JR, Ball DW. The characterization of high-resolution G-banded chromosomes in man. Chromosoma 1978;67:293-307.

${ }^{8}$ Seabright M. A rapid banding technique for human chromosomes. Lancet $1971 ; 2: 971-2$.

9 Arrighi FE, Hsu TC. Localization of heterochromatin in human chromosomes. Cytogenetics 1971;10:81-6.

10 Dutrillaux B, Lejeune J. Sur une nouvelle technique d'analyse du caryotype humain. $C R$ Acad Sci (D) (Paris) $1971 ; 272: 2638-40$.

Requests for reprints to Dr Albert de la Chapelle, Department of Medical Genetics, University of Helsinki, Haartmaninkatu 3, 00290 Helsinki 29, Finland. 